\title{
La reconnaissance juridictionnelle des monnaies virtuelles
}

\author{
Alain Zamaria"
}

\section{Introduction}

Le droit international monétaire est l'une des rares branches du droit international à échapper au phénomène de juridictionnalisation. Dans son cours sur «La Monnaie en Droit International Public » dispensé en 1929 à l'Académie de la Haye, le baron Boris Nolde mentionne dès le chapitre premier « la carence de la doctrine juridique sur la monnaie »1: « la monnaie n'est-elle pas un phénomène essentiellement économique, au sujet duquel le juriste, et à plus forte raison le juriste international, n'ont rien ou presque à dire ? Si le juriste traite la question, ce n'est que de façon accidentelle, on pourrait presque dire en passant, sans jamais en faire le centre de ses études ${ }^{2}{ }^{2}$

Pourrait-on également dire du juge qu'il traite «en passant » la question monétaire ? De prime abord, une telle assertion est contestable. Les effets des décisions monétaires prises par une banque centrale, telles qu'une dépréciation monétaire, ne sont pas dénuées de conséquences juridiques et juridictionnelles, et ce, dans de nombreuses branches du droit. ${ }^{3}$ Les considérations monétaires ne sont pas non plus ignorées du juge, lequel peut anticiper les conséquences monétaires de ses décisions. Le simple fait, pour le juge français, de se référer à « l'évaluation des dégâts $[\ldots$. a à la date où [...] il pouvait être procédé aux travaux destinés à les réparer » signifie une prise en compte de l'inflation lorsque celle-ci dévalue la réparation de dommages matériels. ${ }^{4}$ Enfin, un contentieux judiciaire lié à la monnaie

* Research Fellow à l'Institut Max Planck Luxembourg pour le droit procédural ; Doctorant à l'Université Panthéon-Sorbonne (Paris I).

1 B. Nolde, La monnaie en droit international, 27 RCADI (1968), 247.

2 Ibid., 247.

3 Pour une illustration de certains effets de la dépréciation monétaire en droit administratif, en droit international, en droit pénal, en droit civil et droit du travail et en droit commercial et droit des assurances, voir M. Tancelin et J. R. Garon, Les Effets de la Dépréciation Monétaire Sur les Rapports Juridiques Contractuels, 12 Les Cahiers de droit (1971).

4 Voir Conseil d'Etat, Assemblée, 21 mars 1947, 77529, publié au recueil Lebon. 
peut surgir lorsque celle-ci est l'objet de trafics ou de détournements, ou lorsqu'est créée et diffusée une fausse monnaie. ${ }^{5}$

Pour catégoriser les aspects monétaires qui intéressent le juriste, le baron Nolde différencie les "éléments de droit public » qui incluent "les problèmes $[\ldots]$ de délimitation entre les souverainetés monétaires et de coordination de ces souverainetés ", ${ }^{6}$ ceux liés au "problème de la monnaie de paiement en droit civil ", " questions [qui] ne viennent qu'en second ordre ", ${ }^{7}$ et " les règles pénales destinées à protéger le système monétaire " que l'auteur « laisse de côté ». ${ }^{8}$ Si l'on devait associer chacune de ces catégories à un juge, on pourrait grossièrement associer les éléments de droit civil et de droit pénal aux juridictions internes et les " éléments de droit public » aux juridictions internationales.

Lannée du cours de Boris Nolde, le principe de souveraineté monétaire a été solennellement rappelé par la Cour permanente de justice internationale dans les affaires des emprunts serbes et brésiliens : «C'est un principe généralement admis que tout État a le droit de déterminer lui-même ses monnaies ».9 Qualifié de "position classique des juristes de droit international ", ${ }^{10}$ ce principe a trouvé l'appui des économistes partisans de la théorie étatique de la monnaie, selon lesquels la monnaie est ce qui est créé et défini comme tel par l'État. Cet axiome juridique du monopole étatique de la monnaie peut se voir contredit aussi bien par l'histoire monétaire que par les développements monétaires contemporains.

Alors que l'essentiel de la monnaie actuellement utilisée est conjointement créée par les États et des banques privées, on assiste, comme en écho au projet hayékien de "dénationalisation de la monnaie ", ${ }^{11}$ à un renouveau du "pluralisme » monétaire associé à un mouvement de désintermé-

5 J. M. Darnis, 'Extrait Du Catalogue Des Fonds d'Archives de la Monnaie de Paris', (2010), disponible à http://www.economie.gouv.fr/files/files/directions_services/ca ef/Documents/Archives/Archives_monnaie_Paris/Fonds_ancien/serieO.pdf (dernière visite le 22 septembre 2018).

6 Nolde, supra note 1, 248.

7 Ibid., 248.

8 Ibid., 252.

9 CPIJ, Emprunts Serbes et Brésiliens, 12 juillet 1929, série A, n 20/21.

10 G. Burdeau, L'exercice des compétences monétaires par les États, 212 RCADI (1991), 236.

11 F. A. Hayek, Denationalisation of Money: An Analysis of the Theory and Practice of Concurrent Currencies (1976). 
diation. ${ }^{12}$ Cette désintermédiation est permise par le développement de monnaies qui ne sont pas émises par des banques centrales étatiques, fonctionnent hors du système bancaire, mais remplissent certaines des fonctions traditionnellement associées aux monnaies, soit celles d'instrument d'échange, d'unité de compte et de réserve de valeur.

En retenant une acception extensive de la monnaie, la qualification de monnaie pourrait inclure les "monnaies alternatives " qui visent à renforcer l'économie locale et sont souvent tolérées par les administrations centrales, les programmes de fidélité tels que les Frequent-Flyer Miles attribués par des compagnies aériennes et objets d'une régulation spécifique, ainsi que les monnaies digitales utilisées pour les réseaux sociaux, les jeux vidéo ou les mondes virtuels et qui sont soumises, parfois inadéquatement, à des dispositions juridiques relevant de la propriété intellectuelle. Bien que différents par leur objet et leur origine, ces instruments ont en commun de poser des problèmes de qualification et de remplir une ou plusieurs fonctions monétaires, tout en échappant au système bancaire.

Parmi celles-ci, les monnaies dites virtuelles font l'objet d'un intérêt croissant. Définies dans un rapport de la Banque Centrale Européenne de 2012 comme un type de monnaies numériques non régulées, émises et souvent contrôlées par ses développeurs, et utilisées et acceptées par une communauté virtuelle spécifique, ${ }^{13}$ les monnaies virtuelles doivent être distinguées des monnaies électroniques et des services de paiement en ligne, lesquels sont généralement réglementés ${ }^{14}$ et requièrent l'utilisation de devises officielles.

La dernière-née et la plus célèbre des monnaies virtuelles est le bitcoin, cryptomonnaie apparue en 2009 et dont le fonctionnement est expliqué

12 Le sociologue Nigel Dodd voit dans la « diversification » et "l'homogénéisation » - laquelle se traduit par l'émergence d'instruments financiers globaux, la dollarisation et l'établissement d'unions monétaires - les deux grandes "tendances compensatoires » qui s'observent dans le monde de la monnaie depuis les années 1970, voir 'Bitcoin, Utopianism and the Future of Money - King's Review Magazine, kingsreview.co.uk, 14 March 2015, disponible à http://kingsreview.co.uk/articles/ Bitcoins-utopianism-and-the-future-of-money/ (dernière visite le 22 septembre 2018).

13 European Central Bank, Virtual Currency Schemes, (2012) disponible à http://ww w.ecb.europa.eu/pub/pdf/other/virtualcurrencyschemes201210en.pdf (dernière visite le 22 septembre 2018).

14 Voir par ex. la directive 2007/64/CE du Parlement européen et du Conseil du 13 novembre 2007 concernant les services de paiement dans le marché intérieur, disponible à http://eur-lex.europa.eu/legal-content/FR/TXT/PDF/?uri=CELEX:32007 L0064\&from=FR (dernière visite le 22 septembre 2018). 
dans un livre blanc signé par Satoshi Nakamoto, ${ }^{15}$ pseudonyme de la personne (ou du groupe de personnes) à l'origine de ce système. ${ }^{16}$ Concrétisant l'ambition de désintermédiation de la monnaie, le Bitcoin désigne à la fois une technologie, une unité monétaire (btc) et le réseau de pair-à-pair (peer-to-peer) par lequel ces unités s'échangent. Qualifié de "monnaie d'Internet » comme d' "Internet de la monnaie », ${ }^{17}$ le bitcoin n'a aucune valeur intrinsèque, mais fluctue selon l'offre et la demande (son cours était de 3600 dollars au 11 février 2019). L'originalité du Bitcoin est de ne pas être émis par une banque centrale mais par un réseau décentralisé d'ordinateurs qui vérifient les transactions effectuées. Ces transactions sont regroupées par «bloc » et enregistrées dans une sorte de grand registre sécurisé et accessible à tous les utilisateurs, la Blockchain.

Pour la compréhension de certains des enjeux juridiques posés par le Bitcoin, un aperçu schématique de ces transactions est nécessaire. Les transactions sur le réseau Bitcoin fonctionnent selon la cryptographie asymétrique. Ainsi, chaque utilisateur du Bitcoin possèderait deux clés : une clé (ou adresse) publique et une clé privée, qui sont toutes deux des suites de caractères alphanumériques (1A1zP1eP5QGefi2DMPTfTL5SLmv 7DivfNa est l'adresse publique utilisée pour la première transaction sur le réseau Bitcoin). Une fois créée, l'adresse publique est définitivement intégrée dans la Blockchain et peut être communiquée par une personne souhaitant recevoir des bitcoins, à l'image d'une personne qui communique un relevé d'identité bancaire. La clé privée, quant à elle, doit être précieusement conservée puisqu'elle tient lieu de signature digitale indispensable à tout transfert. Par conséquent, la «propriété » de bitcoins dépend principalement de la possession de la clé privée, laquelle peut être soit stockée physiquement sur un disque dur, soit conservée sur un portefeuille numérique, ce qui suppose l'intervention d'une tierce partie. Le portefeuille numérique est ce qui permet de stocker, recevoir et envoyer des bitcoins. L'image d'un coffre-fort en verre transparent permet de visualiser le principe d'une transaction sur le réseau Bitcoin. À chaque adresse correspond l'un de ces coffres dont l'intérieur peut être observé par tous les passants. En effet, tout

15 "What is needed is an electronic payment system based on cryptographic proof instead of trust, allowing any two willing parties to transact directly with each other without the need for a trusted third party." Cf. S. Nakamoto, Bitcoin: A Peer-toPeer Electronic Cash System, (Bitcoin Project), disponible à https://Bitcoin.org/Bit coin.pdf_(dernière visite le 22 septembre 2018).

16 Voir sur l'identité du créateur du Bitcoin disponible à https://en.Bitcoin.it/wiki/Sa toshi_Nakamoto (dernière visite le 22 septembre 2018).

17 A. Antonopoulos, The Internet of Money (2016). 
utilisateur du réseau Bitcoin peut savoir combien de bitcoins sont détenus à une adresse publique donnée. Le seul moyen de dépenser la somme contenue dans l'un de ces coffres est de signer la transaction via la clé privée qui correspond à celle du coffre. Une transaction consiste à déplacer les bitcoins d'un portefeuille numérique à un autre par le biais d'un message électronique. Une fois la transaction confirmée par le réseau, celle-ci est définitivement gravée dans le marbre de la Blockchain et peut être observée par tous les utilisateurs.

Lémission de bitcoins est déflationniste (le nombre de bitcoins en circulation n'excèdera jamais 21 millions et décroît progressivement) et se fait par un procédé appelé " minage ", comme par analogie à l'extraction des mines d'or. Par ce procédé, les « mineurs » emploient leur matériel informatique pour résoudre des équations complexes permettant de valider et sécuriser les transactions effectuées. En guise de contrepartie, les mineurs perçoivent des bitcoins qui s'ajoutent au grand registre et constituent une sorte de frais sur les transactions confirmées (actuellement une somme de 12,5 bitcoins par « bloc » validé est attribuée aux mineurs).

Les questions que pose ce nouveau type de monnaie dépassent largement le champ juridique et sont également d'ordre technologique (problème d'évolutivité ou scalability) et économique (problème de volatilité et de pérennité de la cryptomonnaie). Que ce système soit légal ou illégal, juridiquement encadré ou affranchi de toute contrainte règlementaire, le Bitcoin est né hors du droit et conçu pour fonctionner sans immixtion du juge ou du législateur.

Dans un ouvrage publié en 1991 et intitulé Order without Law, Robert Ellickson montre de manière provocante, en partant d'une étude sociologique et anthropologique du comté de Shasta aux Etats-Unis, à quel point le droit peut n'avoir aucune incidence sur la vie des gens. D'après l'auteur, pour l'élevage du bétail comme pour obtenir le remboursement d'une dette ou la résolution d'un litige, ce sont bien souvent des normes sociales aussi diverses que « le ragot, le rituel et le culte du héros » qui tiennent lieu de procédure. ${ }^{18}$ C'est donc hors des prétoires que les litiges sont résolus, et souvent selon des règles différentes de celles du droit en vigueur.

L'ordre sans le droit est précisément ce qui est souhaité par les thuriféraires des monnaies virtuelles, en particulier les «techno » ou « crypto-libertariens » désireux de voir la monnaie échapper à tout contrôle du juge ou du législateur. Un aperçu du fonctionnement de ces monnaies permet

18 R. C. Ellickson, Order Without Law: How Neighbors Settle Disputes (1994), 230. 
de constater qu'elles sont, à des degrés divers, conçues pour ne pas être affectées par des dispositions juridiques.

Sans considérer l'ordre monétaire d'un point de vue macroéconomique (lequel concernerait la stabilité des taux d'intérêt, des taux de change et des prix), on pourrait considérer pour la présente étude que la notion recouvre deux des questions juridiques que posait le baron Nolde sur la monnaie : celle de l'ordre face au " problème de la monnaie de paiement en droit civil ", celle de l'ordre assuré par les " règles destinées à protéger le système monétaire ». Quant au problème de la "délimitation entre les souverainetés monétaires ", celui-ci se pose différemment, ces monnaies apparaissant dissociées de la souveraineté. En d'autres termes, l'ordre en matière monétaire concerne, d'une part, l'atteinte des objectifs qui définissent une monnaie dont, au premier rang, la sécurité des transactions pour assurer la fonction d'échange et, d'autre part, l'absence de fraude au sens large, la monnaie ne devant pas être utilisée pour blanchir de l'argent, se soustraire à l'imposition fiscale ou financer des activités illicites.

La reconnaissance juridictionnelle des monnaies virtuelles permet leur élévation au statut de monnaie, ce qui est symboliquement significatif et potentiellement bénéfique aux opérateurs économiques qui l'utilisent. Il existe toutefois un décalage entre la force symbolique de l'État qui reconnaîtrait, par la voie juridictionnelle, une entorse au principe de souveraineté monétaire et la dimension pratique de cette reconnaissance. En effet, si une telle reconnaissance semble avoir l'effet d'une onction du juge (I), elle vise communément la subordination de ces monnaies à des dispositions juridiques liées à la fiscalité et à la sécurité financière (II). Cette finalité (soumettre les monnaies virtuelles à l'ordre juridique) peut apparaître en décalage avec le laissez-faire qui sous-tend l'idéologie des défenseurs de ces monnaies destinées à fonctionner hors du droit.

Bien que principalement consacrée aux décisions américaines portant sur le Bitcoin, la présente étude n'a pas pour objet de refléter l'état du droit sur telle ou telle monnaie virtuelle dans une juridiction donnée. Un tel examen aurait un intérêt limité par la « durée de vie » de la jurisprudence récente. L'étude visera plutôt à livrer un regard critique sur la qualification juridictionnelle des monnaies virtuelles, sur l'impact de telles qualifications sur leur utilisation et, plus largement, sur la manière dont les tribunaux peuvent appréhender des technologies nouvelles dont le fonctionnement suppose, paradoxalement, leur non-ingérence.

Quant au choix de privilégier les décisions américaines, celui-ci s'explique par des raisons d'opportunité : notamment en raison du fédéralisme et de la tradition américaine de défiance à l'égard de l'autorité centrale, les monnaies privées ont prévalu avant la ratification de la Constitution et 
sont restées très répandues jusqu'au début XXe siècle, avant de réapparaittre il y a trois décennies ${ }^{19}$ et d'éveiller un regain d'intérêt avec l'émergence des monnaies virtuelles au tournant du siècle.

\section{L'onction du juge : une reconnaissance nécessaire}

Le traitement des monnaies privées par les juridictions des Etats-Unis illustre l'importance du rôle du juge quant à leur pérennité. En effet, leur continuité semble assurée lorsqu'un juge les reconnaît. À l'inverse, un juge peut interdire leur circulation ou les supprimer, comme ce fut le cas pour plusieurs monnaies privées interdites sous l'impulsion du Département de la justice des Etats-Unis, ${ }^{20}$ non sans un certain retentissement dans un État où la liberté économique et la non-ingérence de l'État fédéral dans l'économie sont sacralisées (A). À rebours de ces exemples, le Bitcoin s'est vu reconnaître le statut de monnaie par divers juges, ce qui a été accueilli par certains partisans de la cryptomonnaie comme leur consécration (B).

\section{A. L'interdiction de monnaies privées centralisées}

Létude de la jurisprudence américaine relative aux monnaies virtuelles nécessite préalablement de considérer le cadre juridique des monnaies privées aux Etats-Unis, la monnaie étant l'objet de dispositions constitutionnelles et de lois fédérales et étatiques.

\section{La légalité contestée des monnaies privées en droit américain}

Pour des raisons historiques et politiques, la légalité des monnaies privées est un sujet sensible aux Etats-Unis, où l'histoire monétaire montre que c'est par le biais de la création et du contrôle monétaires que les pouvoirs du gouvernement fédéral se sont renforcés et que la centralisation s'est réa-

19 L. D. Solomon, Local Currency: A Legal and Policy Analysis, 59 The Kansas Journal of Law \& Public Policy (1996), 81.

20 Le Département de la justice constitue un « département exécutif des États-Unis au siège du gouvernement " (U.S. Code Title $28 \$ 501$ - Executive departmentCode des Etats-Unis), dirigé par le procureur général des Etats-Unis (United States Attorney General). 
lisée dès la fin du XIXe siècle. ${ }^{21}$ C'est pour parachever celle-ci que la Constitution a confié au Congrès le pouvoir d'émettre une monnaie pour la nation et d'en réglementer la valeur, ${ }^{22}$ tandis qu'elle interdit aux Etats de donner cours légal à autre chose que de l'or ou de l'argent. ${ }^{23}$ Toutefois, hormis quelques références au dollar, la monnaie n'est définie nulle part dans la Constitution. En outre, dans le silence de la Constitution, rien ne semble interdire à des personnes privées d'émettre des monnaies métalliques ou de la «monnaie-papier $»^{24} .25$

Bien que le dollar ne soit pas expressément qualifié de monnaie des Etats-Unis, des lois fédérales interdisent la frappe de monnaie à titre privé. Ainsi, une loi votée par le Congrès en 1862 et amendée à quatre reprises, le Stamp Payments Act, conserve depuis cette date une disposition prohibant l'émission de billets, chèques, bordereaux ou jetons qui auraient vocation à circuler comme une monnaie :

Whoever makes, issues, circulates, or pays out any note, check, memorandum, token, or other obligation for a less sum than $\$ 1$, intended to circulate as money or to be received or used in lieu of lawful money of the United States, shall be fined under this title or imprisoned not more than six months, or both. ${ }^{26}$

En même temps que ce texte prohibe l'émission de monnaies privées dont la valeur dépasse 1 dollar, le U.S. Code interdit l'émission de pièces de métal sur le fondement de dispositions relatives à la contrefaçon :

Whoever, except as authorized by law, makes or utters or passes, or attempts to utter or pass, any coins of gold or silver or other metal, or alloys of metals intended for use as current money, whether in the resemblance of coins of the United States or of foreign countries, or of original design, shall be fined under this title or imprisoned not more than five years, or both. ${ }^{27}$

21 Solomon, supra note $19,60$.

22 Article 1, Section 8, Clause 5 of the US Constitution: Congress shall have Power [...] To coin Money, regulate the Value thereof.

23 Article 1, Section 10, Clause 1: No State shall [...] make any Thing but gold and silver Coin a Tender in Payment of Debts.

24 L'expression « monnaie-papier » qualifie les billets de banque qui étaient convertibles en or ou en argent.

25 Solomon, supra note 19,81 .

26 U.S. Code Title $18 \$ 336$ - Issuance of circulating obligations of less than $\$ 1$.

27 U.S. Code Title $18 \$ 486$ - Uttering coins of gold, silver or other metals. 
Une autre disposition interdit la contrefaçon de jetons ou de monnaie-papier. ${ }^{28}$

Ces textes ont servi de fondement à l'inculpation de Bernard von NotHaus, un entrepreneur qui a créé et distribué dès 1998 la monnaie Liberty Dollar par le biais de l'association « National Organization for the Repeal of the Federal Reserve and Internal Revenue Code » (NORFED devenue en 2009 "Liberty Services»). Il s'agissait d'une monnaie physique dont les pièces affichaient une valeur faciale d'un certain montant de l'unité créée et dont la valeur était indexée sur une quantité donnée de certains métaux. Un communiqué issu du bureau du Procureur des Etats-Unis et intitulé "Defendant Convicted of Minting His Own Currency » ${ }^{29}$ avança que l'intention de l'association NORFED était de faire croire que les Liberty Dollars se confondaient avec la monnaie des Etats-Unis ( NORFED's purpose was to mix Liberty Dollars into the current money of the United States »), ce qui pouvait constituer un crime fédéral d'après le Department of Justice (DOJ) des Etats-Unis. Or le communiqué semble lui-même opérer une confusion entre le droit d'émettre une monnaie privée-d'où la mention des dispositions de la Constitution relatives à l'émission de monnaie-et les dispositions relatives à la contrefaçon, bien que celles-ci semblaient inapplicables en l'espèce (« Liberty Dollars cannot be considered to be 'calculated to deceive an honest, sensible and unsuspecting person of ordinary observation and care dealing with a person supposed to be honnest and upright' ${ }^{30}$ ). Les propos du Procureur des Etats-Unis Tompkins lors de l'annonce du verdict (" a unique form of domestic terrorism ", " anti-government activities ») et sa détermination à démanteler les organisations qui émettent ce type de monnaies ( W We are determined to meet these threats through infiltration, disruption, and dismantling of organizations which seek to challenge the legitimacy of our democratic form of govern-

28 U.S. Code Title $18 \$ 491$ - Tokens or paper used as money.

29 Communiqué de presse du 18 mars 2011, "Defendant Convicted of Minting His Own Currency », Federal Bureau of Investigation, Charlotte Division, disponible à https://archives.fbi.gov/archives/charlotte/press-releases/2011/defendant-convicte d-of-minting-his-own-currency (dernière visite le 22 septembre 2018).

30 United States District Court for the Western District of North Carolina, USA v. Bernard von NotHaus, Defendant's supplemental memorandum in support of motions for judgment of acquittal or new trial pursuant to 29 and 33 of the Federal rules of criminal procedure, 25 March 2013, disponible à http://www.gata.org /files/VonNotHausRetrialMotion-03-25-2013.pdf (dernière visite le 22 septembre 2018). 
ment $\gg)^{31}$ confirment que la question de la légalité des monnaies privées demeure ouverte en l'état du droit.

Enfin, on note que des dispositions propres aux Etats fédérés coexistent avec la réglementation fédérale. ${ }^{32}$ Lewis Solomon, auteur d'une étude sur les monnaies locales, jugeait en 1996 que les trois grandes catégories de monnaies privées qu'il considérait à l'époque- le troc, les mécanismes de réduction associés au dollar américain, et les monnaies communautaires détachées du dollar américain »—pouvaient être légalement émises en vertu du droit fédéral et du droit des Etats, à l'exception de celui de la Virginie et de l'Arkansas. ${ }^{33}$

Cette description sommaire de l'arrière-plan juridique permet de comprendre la situation du juge, confronté à un maquis de lois fédérales et étatiques et de réglementations dont il est difficile d'extraire un encadrement juridique compréhensible et stable. Ce contexte est propice à un renforcement du rôle du juge, lequel est d'autant plus important que la reconnaissance de la légalité des monnaies privées est souvent un préalable au développement de leur utilisation et que l'incertitude juridique a pour effet d'inhiber leur croissance économique et de nuire à leur pérennité. ${ }^{34}$

Bien loin d'être indifférent au sort de ces monnaies qui lui échappent, l'État fédéral est intervenu chaque fois qu'une monnaie lui est apparu constituer une menace pour l'ordre public, recourant à leur interdiction le cas échéant. Ainsi, pour faire face aux risques de blanchiment d'argent, de financement du terrorisme, d'achats de biens et de services illégaux, de fraude et d'évasion fiscales, des poursuites judiciaires ont été entreprises par le DOJ et ont abouti à l'interdiction de plusieurs monnaies privées, confirmant que le juge peut avoir un pouvoir de vie et de mort sur cellesci.

\section{L'ascension et la chute de Liberty Reserve}

Parmi les opposants au monopole étatique de la monnaie, certains défendent la concurrence monétaire comme voie d'entrée sur un marché per-

31 Communiqué de presse du 18 mars 2011, supra note 29.

32 Voir par ex Florida v. Espinoza, No F14- 2923, 22nd July 2016, United States, Florida.

33 Solomon, supra note 19, 60.

34 J. J. Doguet, The Nature of the Form: Legal and Regulatory Issues Surrounding the Bitcoin Digital Currency System, 73 Louisiana Law Review (2013), disponible à http://jjdoguet.com/files/Bitcoin.pdf (dernière visite le 22 septembre 2018). 
mettant à des monnaies de circuler parallèlement au dollar. ${ }^{35}$ Au-delà du cercle des partisans de la concurrence monétaire, des spéculateurs, des fraudeurs et des joueurs ont adopté différents types de monnaies virtuelles centralisées qui se sont développées avant l'émergence du Bitcoin. Aussi distingue-t-on parmi ces monnaies centralisées celles utilisées dans des jeux vidéo, notamment Second Life et World of Warcraft, ainsi que des services de paiement en ligne qui consistent à déposer des fonds convertibles à tout moment en or physique (e-gold) ou échangeables contre des monnaies dont la valeur est indexée sur celle de l'once d'or ou de devises officielles (Liberty Reserve). ${ }^{36} \mathrm{Si}$ les monnaies des jeux virtuels ont acquis une importance notable en raison de leur usage, ${ }^{37}$ ces deux modèles de systèmes de paiement en ligne ont été supprimés par le DOJ, suscitant à chaque fois une controverse politique et juridique.

L'e-devise «e-Gold » a été créée par Arthur Budovsky et Vladimir Kats en 1996. Ce service de paiement en ligne consistait à échanger des unités de monnaie numérique présentées comme étant convertibles en métaux précieux, la seule condition pour souscrire à ce service étant de fournir une adresse mail valide (sans obligation de révéler son identité). Des tiers étaient chargés d'échanger des dollars contre la devise numérique pour procéder aux transactions sur les comptes des utilisateurs. Plus de deux millions de comptes ont été ouverts et les transactions auraient atteint un pic de 6 millions de dollars par jour. ${ }^{38}$ Dans le même temps, l'anonymat que permet ce procédé a donné lieu à toute sorte d'activités illicites (blanchiment d'argent, escroquerie, vente de contenus pédopornographiques) qui ont conduit à l'intervention du $\mathrm{DOJ}^{39}$ et à la condamnation des principaux dirigeants à cinq années d'emprisonnement par la suite converties en périodes de probation.

35 L. H. White, "The Troubling Suppression of Competition from Alternative Monies: The Cases of the Liberty Dollar and E-Gold”, 2 Cato Journal 34 (2014), disponible à http://object.cato.org/sites/cato.org/files/serials/files/cato-journal/201 4/5/cato-journal-v 34n2-5.pdf (dernière visite le 22 septembre 2018).

36 Ibid., 283.

37 L'économie du seul jeu Second Life aurait représenté la somme de 500 millions de dollars en 2015, disponible à http://www.investopedia.com/terms/s/second-life-ec onomy.asp (dernière visite le 22 septembre 2018).

38 White, supra note $35,86$.

39 Communiqué de presse du 27 avril 2007, Department of Justice, Digital Currency Business E-Gold Indicted for Money Laundering and Illegal Money Transmitting, disponible à https:/www.justice.gov/archive/opa/pr/2007/April/07_crm_301.html (dernière visite le 22 septembre 2018). 
Pour retenter l'expérience en échappant à la qualification de société de transfert de fonds-soit à celle de "money transmitter » dont les conditions ont changé depuis l'entrée en vigueur du Patriot Act en 2001-et à de nouvelles poursuites du DOJ, Arthur Budovsky décida de fuir les Etats-Unis au cours de sa période de probation afin de lancer un système de paiement similaire au Costa-Rica, Liberty Reserve.

Qualifié de banque du milieu criminel, le système Liberty Reserve permettait la conversion des fonds déposés en «Liberty Reserve Dollars » ou "Liberty Reserve Euros » dont la valeur était indexée sur celle du dollar, de l'euro ou de l'once d'or. Il renforçait par ailleurs l'anonymat des utilisateurs grâce à des intermédiaires réalisant des échanges " en vrac ». Ce système d'échange devait se pérenniser par le prélèvement d'un pourcentage sur chaque transaction. Toutefois, la tentative d'échapper à l'application des lois américaines s'est heurtée à la détermination du DOJ à poursuivre les entreprises de blanchiment d'argent basées à l'étranger, grâce à leur coopération avec divers services secrets étrangers. ${ }^{40}$ Ainsi, dans un communiqué du 28 mai 2013, le DOJ a énuméré les raisons pour lesquelles Liberty Reserve a été condamné :

Liberty Reserve is alleged to have had more than one million users worldwide, including more than 200,000 users in the U.S., who conducted approximately 55 million transactions-virtually all of which were illegal-and laundered more than $\$ 6$ billion in suspected proceeds of crimes including credit card fraud, identity theft, investment fraud, computer hacking, child pornography and narcotics trafficking. ${ }^{41}$

En raison des infractions commises à cause de l'anonymat inhérent à cette monnaie privée, les fondateurs ont été poursuivis et condamnés par le DOJ et la société Liberty Reserve a été liquidée.

Si ces deux services-comme d'autres monnaies virtuelles centralisées plus marginales-ont été interdits, on peut douter qu'ils eurent été viables à long terme. Lanonymat garanti par ces monnaies et les défaillances liées

40 "Liberty Reserve court case marks the dawn of a new era", Brave New Coin, 8 May 2016, disponible à https://bravenewcoin.com/news/liberty-reserve-court-case-mark s-the-dawn-of-a-new-era/ (dernière visite le 22 septembre 2018).

41 Communiqué de presse du 28 mai 2013, Department of Justice, Manhattan U.S. Attorney Announces Charges Against Liberty Reserve, One Of World's Largest Digital Currency Companies, And Seven Of Its Principals And Employees For Allegedly Running A \$6 Billion Money Laundering Scheme, disponible à https:/www.justice.gov/usao-sdny/pr/manhattan-us-attorney-announces-charges-a gainst-liberty-reserve-one-world-s-largest (dernière visite le 22 septembre 2018). 
à l'identification des utilisateurs faisaient qu'il ne pouvait y avoir un système de règlement des différends opérant, par exemple en cas de fraude commise lors d'une transaction. Les caractéristiques novatrices du Bitcoin renouvellent les questions juridiques : le Bitcoin étant par essence virtuel et fonctionnant de manière décentralisée, l'application du droit est rendue plus délicate et la liquidation de ce système de paiement par le DOJ, hautement improbable.

\section{B. La reconnaissance d'une monnaie virtuelle décentralisée}

De nombreuses juridictions nationales ont déjà rendu des décisions dans lesquelles le Bitcoin est mentionné à titre incident, notamment des poursuites pénales liées à des transactions illicites et des litiges impliquant des sociétés qui emploient des bitcoins. De plus rares décisions portent sur la nature du bitcoin et qualifient celui-ci de monnaie, fournissant ainsi de précieux éléments pour leur appréhension juridique.

\section{US Security and Exchange Commission v. Trendon Shavers}

La décision Security and Exchange Commission v. Trendon Shavers ${ }^{42}$ constitue le premier précédent par lequel une juridiction américaine reconnaît explicitement le Bitcoin comme une monnaie. Laffaire illustre un classique schéma de Ponzi : Trendon Shavers, fondateur et dirigeant de BST, une société investissant dans les bitcoins, promettait un taux d'intérêt d'au moins $7 \%$ à ses actionnaires grâce à ses prétendues pratiques d'arbitrage de bitcoins. Or c'était dans son propre porte-monnaie numérique qu'il incluait les bitcoins reçus des investisseurs, constituant ainsi un « fonds de réserve » qui lui permettait de régler les demandes de retraits des investisseurs quand il n'en tirait pas un retour suffisant. Ce faisant, Shavers a commis une fraude portant sur une somme de plus de 60 millions de dollars. Contestant que les investissements en bitcoins s'assimilent à des titres financiers, tels que définis par le Securities Act de 1933 et l'Exchange Act de 1934, le défendeur voulait se prévaloir de l'absence de régulation du Bitcoin pour échapper à ces dispositions. Par une décision du 18 septembre 2014, le juge de la Cour du District du Texas Amos Mazzant a rejeté son argumentation

42 Securities and Exchange Commission v. Trendon T. Shavers and Bitcoin Savings and Trust, Civil Action No. 4:13-CV-416 (2014). 
et qualifié les opérations de "vente de titres ». Trendon Shavers fut condamné à restituer l'équivalent de 40 millions de dollars et à régler 150 000 dollars au titre des sanctions civiles (civil penalties). En juillet 2016, il fut ensuite condamné à 18 mois de prison après avoir plaidé coupable.

On ne peut déduire de la décision que les bitcoins sont des titres financiers. En effet, la question posée à la Cour était de savoir si les investissements de la société BST constituaient de tels titres; elle ne portait donc pas directement sur le Bitcoin, support de ces investissements. La Cour a précisé que les investissements de la société correspondaient à la définition des contrats d'investissement et que ces types de transactions basées sur des monnaies virtuelles étaient assimilables à des titres. Cette interprétation se justifiait au regard des conditions d'existence d'un contrat d'investissement, telles que définies dans les arrêts Howey ${ }^{43}$ et Long v. Shultz Cattle Co. ${ }^{44}$ Dès lors que la société BST a usé de « contrats, transactions» ou d'un " mécanisme impliquant (1) un investissement dans une monnaie, (2) une entreprise commune, (3) avec des profits provenant des efforts d'une tierce partie ${ }^{45}$ les conditions du test étaient remplies pour qualifier l'opération de contrat d'investissement.

Le Bitcoin peut donc être l'objet d'un contrat d'investissement au même titre qu'une monnaie et son commerce peut constituer une " entreprise commune ». Le jugement est également instructif en ce qu'il permet d'évaluer le calcul des dommages causés, le juge devant tenir compte du prix moyen du bitcoin au jour où la fraude a été découverte. Dans d'autres affaires liées à une utilisation du Bitcoin à des fins illicites, des juridictions américaines sont allées plus loin dans l'analyse de la monnaie virtuelle.

\section{L'affaire Silk Road}

Lancé en 2011, le site Silk Road était un marché en ligne accessible sur le Darkweb et permettant la réalisation de transactions illicites en tout anonymat et en ayant exclusivement recours aux bitcoins. Qualifié de "plus sophistiqué et complet des marchés criminels sur Internet $»,{ }^{46}$ le site fut fer-

43 SEC v. W.J. Howey \& Co., 328 U.S. 293 (1946).

44 Long v. Shultz Cattle Co, 881 F.2d 129 (1989).

45 Traduction libre, Securities and Exchange Commission v. Trendon T. Shavers and Bitcoin Savings and Trust, Civil Action No. Civil Action No. 4:13-CV-416.

46 Communiqué de presse du 16 janvier 2014, Department of Justice, U.S. Attorney's Office, Southern District of New York, Manhattan U.S. Attorney Announces Forfeiture Of \$28 Million Worth Of Bitcoins Belonging To Silk Road, disponible 
mé en 2013 par le Federal Bureau of Investigations (FBI) à la suite de ce qui constitue à ce jour la plus importante saisie dans une poursuite pénale liée à l'utilisation de bitcoins. L'affaire Silk Road a donné lieu à deux procès devant la Cour fédérale de Manhattan : la poursuite de Ross William Ulbricht ${ }^{47}$ d'une part, celle de Charlie Shrem et de Robert Faiellaa ${ }^{48}$ d'autre part.

Déjà poursuivi au civil pour la confiscation civile des actifs issus de Silk Road, Ulbricht fut également poursuivi au pénal en tant que créateur, utilisateur et propriétaire du site Silk Road. Il fut inculpé pour 7 chefs d'accusation, dont ceux de conspiration de blanchiment d'argent et de conspiration de trafic de stupéfiants. La saisie des clés privées réquisitionnées a porté sur la somme de 173991 bitcoins (soit l'équivalent de plus de 33 millions de dollars à l'époque). Poursuivis pour des faits similaires à ceux reprochés à Ulricht, Shrem et Faiella ont été condamnés pour blanchiment d'argent et exercice illégal d'une activité de transfert d'argent ( «unlicensed money transmitting business »).

Le premier citait pour sa défense une disposition de l'Internal Revenue Service (IRS), l'autorité fiscale américaine selon laquelle le Bitcoin devait être traité comme un bien aux fins de considération fiscale (" a property for tax purposes $).{ }^{49}$ Les seconds avançaient que le Bitcoin n'était pas une monnaie pour échapper à l'inculpation pour activité de transfert de d'argent non-autorisée.

Considérant dans le cadre de l'affaire Faiella qu'il était bien possible de blanchir de l'argent en recourant au Bitcoin et que celui-ci pouvait être un instrument monétaire, le juge Jed Rakoff en fit une brève description :

[Bitcoin] is digital and has no earthly form; it cannot be put on a shelf and looked at or collected in a nice display case. Its form is digitalbits and bytes that together constitute something of value. ${ }^{50}$

à https://www.justice.gov/usao-sdny/pr/manhattan-us-attorney-announces-forfeitu re-28-million-worth-Bitcoins-belonging-silk (dernière visite le 22 septembre 2018).

47 United States v. Ulbricht, 31 F.Supp.3d 540 (SDNY 2014), disponible à https://case text.com/case/united-states-v-ulbricht-7 (dernière visite le 22 septembre 2018).

48 United States v. Faiella, 39 F.Supp.3d 544 (SDNY 2014), disponible à https://casete xt.com/case/united-states-v-faiella (dernière visite le 22 septembre 2018).

49 I.R.S Notice 2014-21, disponible à https://www.irs.gov/pub/irs-drop/n-14-21.pdf (dernière visite le 22 septembre 2018).

50 Notons qu'un juge de la Cour suprême des Etats-Unis, Stephen Breyer, a été plus loin dans la reconnaissance du Bitcoin comme potentielle monnaie en suggérant dans une opinion dissidente rendue le 21 juin 2018 dans l'affaire Wisconsin Central Ltd. v. United States que le concept de monnaie pouvait évoluer («what we view as 
Certes, les bitcoins sont une monnaie numérique dont la possession dépend de celle d'une clé privée qui se présente sous la forme d'une suite de lettres et de chiffres. Toutefois, on pourrait nuancer ces propos en précisant que la possession d'une clé privée peut être matérialisée. Il suffit en effet d'imprimer ou de noter une clé privée sur une feuille, voire de stocker un portefeuille numérique sur un disque dur, pour que le Bitcoin ait une dimension physique.

D'après un auteur, le fait que le FBI ait pu saisir ("seize ») des bitcoins signifie que le bitcoin n'est pas un bien meuble incorporel et devrait plutôt être qualifié de tangible, ${ }^{51}$ ce qu'on pourrait traduire par «bien meuble corporel » en droit des biens. La justification n'est pas totalement convaincante puisqu'une saisie peut porter sur des biens meubles incorporels. ${ }^{52}$ Cependant, une telle qualification aurait le mérite de simplifier les problèmes de qualification du bitcoin, au moins pour les enjeux de procédure civile. Ainsi, la question de la localisation de bitcoins pourrait trouver une réponse, ceux-ci pouvant être considérés comme étant dans la juridiction dans laquelle se trouve le détenteur-ou la matérialisation-de leur clé privée. Bien que cette approche conduise inéluctablement à des difficultés pratiques, elle semble juridiquement concevable : le droit de propriété exige souvent une attitude du possesseur qui doit, par exemple, veiller à la conservation de son bien ou à sa non-divulgation s'il s'agit d'un droit de propriété intellectuelle.

Toutefois, l'utilisation polymorphe du Bitcoin fait qu'une qualification uniforme de celui-ci n'est ni réaliste, ni souhaitable. Aussi est-ce par opportunité que le juge reconnaît la qualité de monnaie au Bitcoin lorsqu'il juge nécessaire de soumettre son utilisation au droit.

money has changed over time ») et inclure les cryptomonnaies dans son champ (« perhaps one day employees will be paid in Bitcoin or some other type of cryptocurrency ").

51 M. Raskin, "Realm of the Coin: Bitcoin and Civil Procedure", 20 Fordham Journal of Corporate and Financial Law No.4 disponible à https://ssrn.com/abstract=2620 309 (dernière visite le 22 septembre 2018).

52 Pour des exemples de saisies de biens meubles incorporels en droit français, cf. http://www.europe-eje.eu/fiche-thematique/fiche-4-saisie-meubles-incorporels-fich e-juriste-1 (dernière visite le 22 septembre 2018). 


\section{L'emprise du juge : une reconnaissance opportune}

Trois paliers peuvent décrire la latitude de l'État par rapport aux monnaies privées : l'interdiction, l'encadrement et la tolérance. Si certaines monnaies ont été purement et simplement interdites par le DOJ, les tribunaux les reconnaissent plus souvent pour les soumettre au droit, pour des raisons généralement liées à la fiscalité (A) et à la sécurité financière (B).

\section{A. Les enjeux de fiscalité: Skatteverket $v$. Hedqvist}

Le 22 octobre 2015, la Cour de justice de l'Union Européenne a rendu dans l'affaire Skatteverket $v$. Hedqvist ${ }^{53}$ la première décision d'une juridiction supranationale sur une cryptomonnaie. Aussi importante pour les fournisseurs de services qui ont recours aux monnaies virtuelles que pour la qualification du Bitcoin, il est révélateur que cette décision porte sur la taxation des bitcoins et la directive sur la Taxe sur la Valeur Ajoutée $e^{54}$ (TVA). L'engouement que suscitent les questions de fiscalité témoigne en effet de l'intérêt immédiat que représente pour les États la taxation des transactions en bitcoins.

La directive TVA a pour objet d'établir un système commun de taxe sur la valeur ajoutée. En vertu de cette directive, "les livraisons de biens [et] les prestations de services effectuées à titre onéreux sur le territoire d'un État membre par un assujetti agissant en tant que tel [...] sont soumises à TVA. " ${ }^{55}$ Parmi les opérations exclues de son champ d'application, on trouve celles portant sur « les devises, les billets de banque et les monnaies qui sont des moyens de paiement légaux ». L'affaire Skatteverket renseigne sur la nature des monnaies virtuelles en posant la question de l'applicabilité de cette disposition aux opérations portant sur les bitcoins.

En l'espèce, un citoyen suédois souhaitait fournir, par l'intermédiaire d'une société, des services d'échange de devises traditionnelles contre des bitcoins, et vice-versa. Pour ce faire, il avait demandé à la commission suédoise de droit fiscal si la TVA devait être acquittée lors de l'achat et de la vente de Bitcoins. Assimilant le Bitcoin à « un moyen de paiement utilisé

53 Hedqvist, cas C-264/14, arrêt du 22 octobre 2015, ECLI:EU:C:2015:718.

54 Directive 2006/112/CE du Conseil, du 28 novembre 2006, relative au système commun de taxe sur la valeur ajoutée (JO L347, p.1).

55 Ibid., art. 2. Voir aussi Communiqué de presse no 128/15, affaire C-264/14, Skatteverket/ David Hedqvist, Cour de justice de l'Union européenne, Luxembourg. 
de manière analogue aux moyens de paiement légaux ", la commission avait jugé que les opérations devaient être exonérées de la TVA. ${ }^{56}$ L'autorité fiscale suédoise en charge de la collecte des impôts nationaux, la Skatteverket, contesta l'interprétation de la commission devant la Cour administrative suprême, le Högsta förvaltningsdomstolen. Cette juridiction décida de surseoir à statuer et de poser à la CJUE la question de savoir si les opérations d'échange de devises virtuelles contre des devises traditionnelles et vice-versa constituaient des prestations de services effectuées à titre onéreux en vertu de l'article 2 (1) de la directive TVA et, dans l'affirmative, si ces opérations étaient exonérées de cette taxe.

La Cour a répondu par l'affirmative aux deux questions. Concernant la première, le fait que ces opérations avaient consisté à échanger différents moyens de paiement et qu'il existait un lien entre le service rendu et la contre-valeur reçue, soit une marge entre le prix d'achat des devises et le prix de revente, permet de déduire qu'il s'agissait bien de " prestations de services effectuées à titre onéreux " telles qu'énoncées dans la directive. Concernant la seconde, la Cour a jugé que ces opérations portaient sur une monnaie, qu'il en découle qu'elles entraient dans le champ de l'exemption prévue à l'article 135 (1) de la directive pour « les devises, les billets de banque et les monnaies qui sont des moyens de paiement légaux » et qu'elles devaient donc être exonérées de TVA.

Par cette décision, la Cour a ouvert la voie à une appréhension juridique des monnaies virtuelles en tenant compte de leur spécificité. Pour mettre en avant le vide réglementaire autour du Bitcoin, ${ }^{57}$ la juridiction de renvoi reprenait les analyses d'un rapport de la Banque centrale européenne (BCE) de 2012 sur les monnaies virtuelles :

The instability of virtual currency schemes can be explained by one of the most critical aspects mentioned earlier, i.e. the lack of a proper legal basis for virtual currency schemes. ${ }^{58}$

Dans son rapport, la BCE justifiait par la manière dont ces monnaies virtuelles sont émises le rejet de la qualification de monnaie électronique et de l'applicabilité des directives 2009/110/EC et 2007/64/CE relatives respectivement aux monnaies électroniques et aux services de paiement. ${ }^{59} \mathrm{Au}$-delà de ses conséquences fiscales, cette décision est donc intéressante pour

56 Hedqvist, supra note 52, point 17.

57 Ibid., à point 12.

58 Virtual Currency Schemes, supra note 13, 42.

59 Ibid., 16, 17. 
l'analyse qui est faite du Bitcoin et pour son éclairage sur le système européen d'imposition indirecte.

L'une des caractéristiques du système européen de TVA est d'éviter que le simple fait de payer une somme d'argent ne constitue un fait générateur d'imposition. Sauf à imaginer que l'on consomme une monnaie de même que certains moyens de paiement consommables comme l'or ou les cigarettes, ${ }^{60}$ aucune valeur ajoutée n'est créée lors d'un échange d'argent, ce qui justifie l'absence d'imposition à la TVA. ${ }^{61}$ Ce principe fut énoncé dans les arrêts Mirror Group ${ }^{62}$ et Fitzgerald, ${ }^{63}$ puis par l'arrêt BUPA Hospitals, ${ }^{64}$ dans lequel la CJUE a affirmé que « ce sont les livraisons de biens et les prestations de services qui sont soumises à la TVA et non les paiements effectués en contrepartie de celles-ci. » Dans l'affaire First National Bank of Chicago, ${ }^{65}$ la Cour avait déjà admis que des transactions monétaires par lesquelles une banque échange une devise nationale contre des devises étrangères sont exonérées de TVA, tout en considérant que la différence entre l'offre et la demande, le spread, constitue un enrichissement du banquier pour les transactions effectuées. Dans cette espèce, le gouvernement du Royaume-Uni jugeait que, «faute de contrepartie, une opération de change exécutée sans prélever de commission ou de frais bancaires ne constitu[ait] pas une livraison de biens ou une prestation de services au sens de la sixième directive, mais n'[était] qu'un simple échange de moyens de paiement. "66 L'argument selon lequel il s'agissait d'un "simple échange de moyens de paiement » qui à ce titre ne devait pas être soumis à la TVA avait été implicitement ${ }^{67}$ suivi par la Cour. Dans ses conclusions pour l'affaire Hedqvist, l'avocat général Juliane Kokott s'est explicitement référé à l'arrêt First National Bank of Chicago et a jugé que les opérations de change en question étaient bien « des prestations de services à titre onéreux au sens de l'article 2, point 1, de la directive $»{ }^{68}$ La Cour exempterait donc de TVA toute activité visant

60 Hedqvist, supra note 52, conclusions de l'avocat général J. Kokott, point 14.

61 R. Wolf, Virtual Currencies, M-Payments and VAT: Ready for the Future?, in G. Gimigliano (ed.), Bitcoin and Mobile Payments, Constructing a European Union Framework (2016), 236.

62 Mirror Group, cas C-409/98, arrêt du 9 octobre 2001, ECLI:EU:C:2001:524.

63 Cantor-Fitzgerald, cas C-108/99, arrêt du 9 octobre 2001, ECLI:EU:C:2001:526.

64 BUPA Hospitals and Goldsborough Developments, cas C-419/02, arrêt du 21 février 2006, ECLI:EU:C:2006:122.

65 First National Bank of Chicago, cas C-172/96, arrêt du 14 juillet 1998, ECLI:EU:C:1998:354.

66 Ibid., à point 24.

67 Wolf, supra note 60, 237.

68 Hedqvist, supra note 52, conclusions de l'avocat général J. Kokott, point 18. 
un échange de moyens de paiement qui n'aurait d'autre finalité que celuici, y compris dans le cas d'un commerce visant à échanger des « devises traditionnelles » contre des « devises virtuelles ».

A priori, l'argument semble convaincant. En effet, pourquoi le Bitcoin devrait-il être traité différemment d'une autre devise ${ }^{69}$ dès lors qu'il peut tout autant servir de moyen de paiement ? N'y voyant aucune objection, l'avocat général assimile les bitcoins aux " purs moyens de paiement, légaux ou autres, tels que les bons d'échange d'une valeur nominale, ou l'acquisition de " points » utilisables ensuite dans des hôtels ou des résidences, » lesquels sont tous traités par la Cour «de la même manière, dans la mesure où elle ne voit pas non plus dans le transfert de [ces] moyens de paiement $[\ldots]$ des opérations imposables. ${ }^{70}$ Ces « purs » moyens de paiement " autres » que légaux correspondent en partie à ce que l'on a qualifié de monnaies privées. Ces dernières étant déjà en train de proliférer grâce au progrès technologique, on peut penser que la Cour de Justice traitera indistinctement les opérations de change entre les "purs moyens de paiement ", que ceux-ci soient légaux ou non, en leur faisant tous bénéficier de l'exemption prévue dans la directive TVA.

Toutefois, il est réducteur de définir le Bitcoin comme un " pur » moyen de paiement, comme l'a fait l'avocat général par ces propos : « [s]elon les constatations de la juridiction de renvoi, les bitcoins constituent également un pur moyen de paiement. Leur possession n'a pas d'autre finalité que de les réutiliser comme moyen de paiement à un moment quelconque. ${ }^{71} S^{\prime}$ il est vrai que le Bitcoin sert de moyen de paiement, il remplit d'autres fonctions classiquement attribuées à une monnaie, en particulier celle de réserve de valeur-certes encore volatile ${ }^{72}$ - et, à un moindre degré, celle d'unité de compte. ${ }^{73}$ De plus, comme cela a été vu, le Bitcoin est à la fois une monnaie, un réseau d'échange de pair-à-pair et une technologie.

Bitcoin, the technology, is finding an increasing quantity of applications beyond its prototypical use case. Many of these applications in-

69 À ce propos, la Cour qualifie le Bitcoin de « devise virtuelle à flux bidirectionnel ».

70 Hedqvist, supra note 52, conclusions de l'avocat général J. Kokott, point 16.

71 Ibid., point 17.

72 W. J. Luther, 'Decline in the Volatility of Bitcoin', Sound Money Project, 25 May 2016, disponible à http://soundmoneyproject.org/2016/05/decline-in-the-volatility -of-Bitcoin/ (dernière visite le 22 septembre 2018).

73 Si la dimension d'unité de compte peut sembler négligeable, on peut toutefois noter que le Bitcoin sert régulièrement d'étalon pour les transactions en cryptomonnaies. 
volve the inscription and communication of blockchain messages, which is all a "Bitcoin transaction" really is: communication. ${ }^{74}$

En effet, les technologies à la base du Bitcoin (notamment la blockchain, la cryptographie asymétrique et le système de validation algorithmique dit "proof-of-work») ouvrent la voie à de nombreuses utilisations possibles. En permettant de retracer l'ensemble des transactions effectuées depuis l'origine et de vérifier ainsi la véracité des informations utilisées (fonction de «truth proving »), ces technologies peuvent être utilisées pour voter en ligne, délivrer des diplômes, établir un registre foncier ou encore transformer le système des chambres de compensation. Une définition holiste du Bitcoin comme pur instrument de paiement-en vertu de laquelle la monnaie serait réduite à son acception de droit privé-rendrait donc son encadrement juridique limité au regard de ses potentialités. ${ }^{75}$

Bien que la fonction de monnaie soit encore première, on peut donc partager l'avis selon lequel, au vu des nombreuses autres fonctions permises par les technologies du Bitcoin, certains de ses usages pourraient être l'objet d'une exonération de TVA tandis que d'autres devraient ne pas pouvoir en bénéficier. ${ }^{76}$

En conclusion, comme toute monnaie, le Bitcoin ne peut être réduit à un instrument de paiement. Il n'est pas non plus réductible à sa seule vocation monétaire. Par ce double raccourci, la Cour méconnait donc le Bitcoin alors même que cette décision est paradoxalement présentée comme sa plus importante consécration juridictionnelle. Ce décalage révèle l'ambiguiité inhérente à la reconnaissance juridictionnelle des monnaies privées : l'objet de ces décisions n'est pas d'appréhender juridiquement ces objets mais d'en retenir une qualification correspondant aux buts recherchés. En l'espèce, le but recherché est celui de la pertinence fiscale. Si certaines monnaies virtuelles peuvent fonctionner sans État, elles peuvent viser à se soustraire à certains impératifs du droit fiscal. Il incombe donc au le juge de les reconnaître pour mieux les soumettre au droit.

74 E. Voorhees, "The Importance of Bitcoin Not Being Money," moneyandstate.com, 8 August 2016, disponible à http://moneyandstate.com/the-importance-of-Bitcoinnot-being-money/ (dernière visite le 22 septembre 2018).

75 Ibid.

76 J. Maupin, "The ECJ's First Bitcoin Decision: Right Outcome, Wrong Reasons?”, VerfBlog, 4 November 2015, disponible à http://verfassungsblog.de/the-ecjs-first-B itcoin-decision-right-outcome-wrong-reasons/ (dernière visite le 22 septembre 2018). 


\section{B. Les enjeux de sécurité financière : State of Florida v. Espinoza}

Une affaire relative à une accusation de blanchiment d'argent, State of Florida $v$. Espinoza,${ }^{77}$ met en relief le lien entre la qualification du Bitcoin et les enjeux de sécurité financière. Dans cette espèce, Michell Espinoza, un particulier vendant des bitcoins contre des espèces, avait accepté d'en vendre à un agent infiltré de la police californienne. L'agent infiltré prétendait vouloir échanger des bitcoins contre des cartes de crédit volées. Espinoza accepta de réaliser plusieurs transactions jusqu'à ce qu'il fût arrêté après avoir refusé une transaction pour un montant de 30000 dollars. Espinoza fut ensuite poursuivi par un tribunal de l'Etat de Floride pour violation de règlementations locales relatives aux entreprises de services monétaires ( money service business ") et au blanchiment d'argent. Au moment où les faits ont été commis, l'État de Floride n'avait ni légiféré, ni émis des lignes directrices sur les monnaies virtuelles. ${ }^{78}$ Par une décision du 22 juillet 2016, la Cour a rejeté le chef d'accusation relatif à l'absence d'autorisation de transmettre de l'argent et jugé que le simple fait de vendre des bitcoins à une personne qui aurait l'intention-fût-elle clairement établie au moment de la transaction-de les utiliser à des fins criminelles ne pouvait servir de fondement à l'accusation de blanchiment d'argent. Cette décision, qui a fait l'objet d'un appel, contraste avec une jurisprudence qui tendait à assimiler le Bitcoin à un instrument monétaire.

Le premier chef d'accusation portait sur l'exercice non-autorisé de services monétaires. La réglementation de l'Etat de Floride qualifie de «money services business " toute personne "who acts as a payment instrument seller, foreign currency exchanger, check casher, or money transmitter $",{ }^{79}$ et requiert un permis d'exercer cette activité. ${ }^{80}$ En l'espèce, l'Etat de Floride accusait le défendeur d'exercer illégalement l'activité de transfert d'argent ( « money transmitter») puis, par un glissement sémantique opéré lors de la dernière plaidoirie, celle de vendeur d'instruments de paiement (« payment instrument seller»).

77 The State of Florida v. Michell Abner Espinoza, Criminal Division Case No. F142923, (Fla. 11th Cir. Ct. 2016).

78 Depuis le $1^{\text {er }}$ juillet 2017, l'Etat de Floride intègre les monnaies virtuelles parmi les instruments monétaires cités dans sa loi sur le blanchiment d'argent, voir House Bill 1379, disponible à https://www.flsenate.gov/Session/Bill/2017/1379 (dernière visite le 22 septembre 2018).

79 Fla. Stat. Section 560.103(22).

80 Fla. Stat. Section 560.125. 
Reprenant point par point les arguments de l'Etat de Floride, la juge Teresa Pooler a, dans un premier temps, considéré que la vente de bitcoins à un agent infiltré ne constituait pas une transmission d'argent. La Cour a jugé que la transmission (" to transmit») supposerait que l'on " envoie ou transfere (une chose) d'une personne ou d'un endroit à un(e) autre », ${ }^{81}$ comme ce fut jugé pour un cas impliquant la société spécialisée dans le transfert d'argent (« money transmitting business ») Western Union. ${ }^{82}$ Dans la mesure où Espinoza n'a pas agi en tant qu'intermédiaire (" middleman ») et n'a fait que vendre des bitcoins en cherchant un profit, la Cour a refusé de le qualifier de "transmetteur ». En effet, pour recevoir la qualification de " money transmitting business ", un individu doit prélever des frais sur les transactions, ce que, selon la juge, le défendeur n’avait pas fait puisqu'il réalisait un profit en vendant les bitcoins pour un montant supérieur à leur prix d'achat. La juge qualifia donc le Bitcoin de "property » (ce qu'on traduirait par «bien meuble ») vendue à titre personnel : "The Defendant was selling his personal property. "

Dans un second temps, la juge a rejeté l'argument selon lequel Espinoza était un vendeur d'instruments de paiement. La réglementation de l'Etat de Floride qualifiait d' "instruments de paiement » les instruments suivants : " check, draft, warrant, money order, travelers' check, electronic instrument, or other instrument, payment of money, or monetary value whether or not negotiable $»{ }^{83}$ En refusant d'assimiler le Bitcoin à l'un de ces instruments, la juge a convenu que le Bitcoin ne constituait ni une monnaie, ni un instrument de paiement, ni même une valeur monétaire ( monetary value »). Pourtant, on aurait pu considérer que les termes employés, en particulier la référence à la valeur monétaire, signifiaient que le législateur voulait que les instruments de paiement visés ne soient pas limités aux seules devises officielles.

Concernant l'allégation relative au blanchiment d'argent, la juge n'a pas reconnu une intention de promouvoir ( intent to promote») une attitude criminelle et a qualifié de "vague » le terme promouvoir, au terme d'une étude linguistique sur le sens des mots "promote », « incite » et « encourage ». La Cour a ensuite critiqué la démarche poursuivie par le Ministère public en jugeant que le défendeur avait simplement accepté de vendre des bitcoins à un détective qui voulait le faire condamner :

81 Cf. définition citée dans la décision : " "transmit" means "to send or transfer (a thing) from one person or place to another". Black's Law Dictionary. $\left(10^{\text {th }}\right.$ ed. 2014)».

82 U.S. v. Elfgeeh, 515 F.3d 100, 108 (2d Cir. 2008).

83 Fla. Stat. Section 560.103(29). 
$[\mathrm{I}] \mathrm{s}$ it criminal activity for a person merely to sell their property to another when the buyer describes a nefarious reason for wanting the property? [...] There is unquestionably no evidence that the Defendant did anything wrong, other than sell his Bitcoin to an investigator who wanted to make a case.

L'argumentation de la juge est critiquable à plusieurs égards : le fait que certaines définitions soient reprises de dictionnaires usuels et non d'un dictionnaire juridique, la distinction opérée entre frais et profits qu'aurait perçus le défendeur ${ }^{84}$ et, surtout, la manière dont est qualifié le Bitcoin au regard de la réglementation relative aux services de transfert d'argent peuvent être longuement discutés. ${ }^{85}$ À propos de la qualification du Bitcoin, la Cour a jugé que cette question relevait de l'économie plus que du droit, en insinuant qu'elle n'était pas l'institution la mieux placée pour opérer une telle qualification : "This court is not an expert on economics ». Sans que la juge ne renonce pour autant à qualifier le Bitcoin, cet aveu se mêlait à une forme d'hommage au sens commun : " however, it is very clear, even to someone with limited knowledge in the area, that Bitcoin has a long way to go before it is the equivalent of money ». La Cour a ensuite repris l'analyse de l'IRS, l'autorité fiscale qui a qualifié le Bitcoin de propriété aux fins de taxation. Ce faisant, elle a pu écarter le chef d'inculpation relatif au blanchiment d'argent: " This Court is unwilling to punish a man for selling his property to another. "

Si l'on peut approuver la juge lorsqu'elle affirme que le Bitcoin ne peut s'échanger contre des biens aussi facilement qu'une monnaie qui a cours légal et qu'il est donc encore loin de pouvoir être considéré comme l'équivalent d'une devise, d'autres arguments sont plus contestables. Après avoir mentionné les limites du Bitcoin comme moyen d'échange, la Cour juge que la volatilité du Bitcoin nuirait à sa fonction de " réserve de valeur » : "With such volatility they have a limited ability to act as a store of value, another important attribute of money. » L'argument est discutable, l'absence de volatilité n'étant pas une condition d'existence d'une monnaie. En

84 Dans la mesure où le défendeur perçoit une somme à chaque transaction, indépendamment du prix d'achat et de revente des bitcoins, la qualification de « frais » semble mieux convenir que celle de "profit », le profit étant lié aux investissements réalisés et non à la seule activité d'échange.

85 A. Caffarone, M. Holzer, "Ev'ry American Experiment Sets a Precedent': Why One Florida State Court's Bitcoin Opinion is Everyone's Business”, Hofstra Law Journal of International Business \& Law (2017); Hofstra Univ. Legal Studies Research Paper No. 2017-03. Available at SSRN: disponible à https://ssrn.com/abstra $\mathrm{ct}=2897727$ (dernière visite le 22 septembre 2018). 
outre, on peut noter que l'argument selon lequel le Bitcoin n'est pas une réserve de valeur est contre-intuitif au vu du nombre de personnes qui se sont enrichies par ce biais.

L'absence de convertibilité du Bitcoin est également présentée comme une lacune du Bitcoin : "Bitcoins are not backed by anything. They are certainly not tangible wealth and cannot be hidden under a mattress like cash and gold bars. » Toutefois, la nature intangible du Bitcoin et l'absence de convertibilité ne devraient pas rendre la qualification de monnaie inopérante. Non seulement les devises officielles ne sont pas convertibles en or ou en argent mais elles peuvent également être intangibles, comme dans le cas des dépôts bancaires. Enfin, il est surprenant de refuser la qualification de monnaie pour le Bitcoin mais de présenter des lingots d'or comme une monnaie au même titre que le cash, alors que l'or est généralement assimilé à une commodité (" commodity») et soumis à un régime juridique et fiscal particulier.

Au prisme de ces approximations, on peut se demander si le choix de qualifier le Bitcoin de bien (ou de " property ») plutôt que d'instrument de paiement ne résulte pas des circonstances de l'espèce. Concernant le blanchiment d'argent, la Cour s'attachait à critiquer l'argument selon lequel le défendeur aurait eu l'intention de promouvoir une action illégale. Le refus de qualifier le Bitcoin comme monnaie aurait donc servi de simple argument additionnel pour rendre infondée l'accusation de blanchiment d'argent. En effet, on peut penser que la même décision aurait été rendue si le Bitcoin avait été qualifié d'instrument monétaire, la qualification monétaire n'ayant d'incidence qu'en ce qui concerne l'accusation relative au transfert d'argent non-autorisé. Sur ce second point, le refus de qualifier le Bitcoin de monnaie semble dû à la manière dont l'activité du défendeur est perçue par la juge. En effet, celle-ci assimile l'achat de Bitcoins à une activité de trading ( The Defendant purchases Bitcoin low and sells them high, the equivalent of a day trader in the stock market, presumably intending to make a profit ») et non à un service monétaire ou à une pratique de conversion de monnaies.

Si la Cour a considéré que le Bitcoin n'était pas un instrument monétaire, elle a néanmoins jugé qu'il s'agissait bien de transactions financières puisque le défendeur échangeait ses biens meubles (" his property») contre des espèces, lesquels constituent un instrument monétaire. Or une transaction financière est définie par le législateur de l'Etat de Floride comme une transaction impliquant le mouvement de fonds par transfert ou par d'autres moyens, ou impliquant un ou plusieurs instruments monétaires 
qui affectent d'une manière ou d'une autre le commerce. ${ }^{86} \mathrm{La}$ Cour ignore donc la première partie de la définition en étant silencieuse sur la qualification de fonds, bien que cette qualification ait été retenue pour le Bitcoin dans l'arrêt Ulbricht ${ }^{87}$ et, plus récemment, dans l'arrêt Murgio ${ }^{88}$ qui portait sur la réglementation fédérale relative aux entreprises de transmission de fonds. ${ }^{89}$

Certes, la portée de la décision semble limitée : rendue par un tribunal de l'Etat de Floride qui applique des dispositions locales, cette décision n'a pas valeur de précédent dans les autres Etats ou au niveau fédéral, ${ }^{90}$ de même que la décision SEC $v$. Trendon Shavers. Toutefois, d'autres tribunaux américains peuvent suivre la Cour de Floride en refusant de qualifier le Bitcoin de valeur monétaire et de l'assujettir aux dispositions relatives aux entreprises de services monétaires. ${ }^{91}$ Par ailleurs, les décisions Espinoza et Shavers illustrent bien l'ambiguïté propre à la reconnaissance juridictionnelle des cryptomonnaies : à chaque fois, la défense avance que le Bitcoin ne peut être qualifié de monnaie, même lorsqu'il est utilisé comme telle en pratique. Paradoxalement, la non-reconnaissance du Bitcoin par la Cour de Floride peut donc servir la cryptomonnaie, en renforçant l'idée selon laquelle le Bitcoin peut être utilisé comme monnaie sans être l'objet d'entraves juridiques.

Les décisions considérées montrent que le juge préfere sacrifier la technicité de définitions du Bitcoin au profit du bon sens. La méconnaissance des subtilités liées à cette monnaie n'est pas un problème en soi : on attend

86 Fla. Stat. Section 560.103 (22): " "Financial transaction" means a transaction involving the movement of funds by wire or other means or involving one or more monetary instruments, which in any way or degree affects commerce [...] ».

87 United States v. Ulbricht, 31 F.Supp.3d 570 "Put simply, "funds" can be used to pay for things in the colloquial sense. Bitcoins can be either used directly to pay for certain things or can act as a medium of exchange and be converted into a currency which can pay for things. ".

88 United States v. Murgio, No. 15-cr-769, 2016 WL 5107128, (S.D.N.Y. Sep. 19, 2016), 4-10.

89 U.S. Code Title $18 \$ 1960$.

90 S. D. Palley, "Why Florida's 'Bitcoin Isn't money' Ruling Could Have Limited Impact," CoinDesk, 28 July 2016, disponible à http://www.coindesk.com/florida-Bitc oin-money-legal-system/ (dernière visite le 22 septembre 2018).

91 Il semble cependant que les juridictions américaines s'orientent vers une interprétation des dispositions relatives aux entreprises de services monétaires qui engloberait l'utilisation des bitcoins. Ainsi, une ordonnance rendue le $1^{\text {er }}$ février 2019 par le Southern Division of the District Court for the Eastern District of Michigan dans l'affaire United States v. Stetkiw 18-20579, E.D. Mich., 2/1/19 établit que : «Bitcoin qualifies as "money" and "funds" under [18 U.S.C.] \$1960. » 
du juge la résolution d'un litige et non l'explication du fonctionnement d'un mécanisme qui se présente à lui. Or le juge semble généralement qualifier le Bitcoin en fonction des seuls impératifs juridiques qu'il veut faire prévaloir, selon des considérations d'opportunité. Les qualifications juridictionnelles varient donc d'une juridiction à une autre de même qu'au niveau des autorités de régulation, au détriment de la cohérence du droit applicable aux monnaies virtuelles. On pourrait expliquer cet éclatement du droit applicable au Bitcoin par le fait que son utilisation polymorphe le rend compatible avec des qualifications différentes, voire opposées.

Des défenseurs des monnaies virtuelles ont demandé que les régulateurs adoptent une approche attentiste par rapport aux cryptomonnaies et ne cèdent pas à la tentation du " classificationnisme bureaucratique.$^{92} \mathrm{Le}$ " classificationnisme " est l'attitude de ceux qui tendent soit à créer de nouvelles catégories juridiques répondant à l'inadéquation entre une activité et les catégories juridiques existantes, soit à tout ramener à la dichotomie entre le légal et l'illégal, comme l'ont fait de nombreux États qui ont choisi d'interdire l'utilisation du Bitcoin. Juridiquement, le Bitcoin n'est ni une devise officielle, ni une monnaie électronique, ${ }^{93}$ ni un titre mobilier. Certains États en ont déduit son illégalité au regard du droit en vigueur. Une telle démarche, surtout lorsqu'elle se concrétise par la mise en place d'une incrimination pénale, risque d'être liberticide et de n'avoir que peu d'incidence pour empêcher ses usages illicites. Aussi est-il préférable que les juges adaptent les catégories juridiques existantes à l'utilisation qui est faite des monnaies virtuelles et des technologies émergentes et que l'on repense les relations entre l'ordre juridique et l'ordre numérique. En effet ces deux ordres peuvent aussi bien s'opposer que se compléter ou s'aligner. Il convient donc de toujours considérer dans quelle mesure et à quelle fin le second s'affranchit du premier.

\section{Conclusion}

Provenant de la déconnexion entre l'ordre numérique et l'ordre juridique, les enjeux juridiques liés au Bitcoin peuvent être considérés à l'aune de ceux qui se sont posés plus tôt pour la gouvernance d'Internet. Comme à l'époque du développement d'Internet, le juge et le législateur se trouvent

92 K. S. Graf, Are Bitcoins Ownable?: Property Rights, IP Wrongs and Legal-Theory Implications (2015), 5.

93 Virtual Currency Schemes, supra note 13. 
confrontés à un système dont le fonctionnement est anarchique par essence. L'ingénieur et activiste Timothy May avait déjà avancé en 1992 le concept de "crypto-anarchisme » pour décrire la primauté et la " préférence des solutions technologiques par rapport aux solutions juridiques ».94 Lawrence Lessig, l'un des pionniers de ce qui est devenu le cyber-droit, imaginait quant à lui que le code informatique pourrait tenir lieu de droit, selon la célèbre expression "Code is law ».

Par analogie au droit d'Internet, on pourrait désormais se demander si le Protocole Bitcoin ne pourrait pas tenir lieu de droit applicable et justifier l'expression «Law is Protocol ", dans la mesure où la cryptographie remplacerait plusieurs institutions fondamentales, telles que le juge lui-même. Ainsi, le Protocole représente la Constitution du Bitcoin et définit la politique monétaire en énonçant qui peut émettre la monnaie (non pas le Congrès comme pour le dollar américain mais tous les utilisateurs du réseau), comment progresse la masse monétaire (une importante « loi » algorithmique du Bitcoin énonce le rythme auquel augmente l'offre monétaire jusqu'à une limite maximale de 21 millions de Bitcoins en circulation) et comment sanctionner l'utilisateur qui tenterait de falsifier le grand registre (en ignorant cette tentative). ${ }^{95}$ D'autres caractéristiques, telles que les comptes multi-signatures, permettent même d'intégrer au système Bitcoin un crypto-droit de la consommation susceptible de régler certains problèmes liés à l'irrévocabilité des transactions. En résumé, on pourrait parler d'un système qui incorpore son propre droit ( law unto itself ${ }^{96}$ ) et rend toute intervention du juge ou du législateur dispensable.

La conséquence des qualifications juridictionnelles divergentes des monnaies virtuelles est une impression générale de confusion : le Bitcoin peut être une monnaie, un fonds, une commodité, ${ }^{97}$ un bien meuble, voire une catégorie à part. Or ces catégories juridiques sont des constructions hu-

94 T. C. May, The Crypto Anarchist Manifesto, 22 November 1992, disponible à http://www.activism.net/cypherpunk/crypto-anarchy.html (dernière visite le 22 septembre 2018).

95 S. Jeong, "The Bitcoin Protocol as Law, and the Politics of a Stateless Currency," SSRN (2013), disponible à https://papers.ssrn.com/sol3/papers.cfm?abstract_id=22 94124 (dernière visite le 22 septembre 2018), 28.

96 Graf, supra note $90,9$.

97 Pour la U.S. Commodity Futures Trading Commission (CFTC), le Bitcoin peut en revanche être qualifié de "commodity " sujette au Commodity Exchange Act, voir communiqué de presse du 17 septembre 2015, CFTC Orders Bitcoin Options Trading Platform Operator and its CEO to Cease Illegally Offering Bitcoin Options and to Cease Operating a Facility for Trading or Processing of Swaps without Registering, disponible à http://www.cftc.gov/PressRoom/PressReleases/pr723 
maines, adaptables au gré des utilisations que l'on fait des nouvelles technologies. Même si les technologies qui fondent le Bitcoin peuvent prévenir des différends survenant dans les rapports privés, les premiers litiges résultant des frottements entre l'ordre juridique et l'ordre numérique conduisent à douter que l'immixtion du juge puisse être évitée. À l'inverse, c'est même à l'émergence d'un nouveau contentieux monétaire que l'on pourrait s'attendre.

1-15 (dernière visite le 22 septembre 2018). La CFTC a réitéré sa position le 3 octobre 2018 en s'appuyant sur une décision rendue le 26 septembre 2018 par le United States District Court of Massachusetts dans l'affaire Commodity Futures Trading Commission v. My Big Coin Pay, Inc. et al. 
